\title{
F-type Pyocins are Diverse Non-Contractile Phage Tail-Like Weapons for Killing Pseudomonas aeruginosa
}

Senjuti Saha ${ }^{\mathrm{a}, \mathrm{c}}$, Chidozie D. Ojobor ${ }^{\mathrm{a}}$, Erik Mackinnon ${ }^{\mathrm{a}}$, Olesia I. North ${ }^{\mathrm{b}}$, Joseph BondyDenomy $^{\text {d }}$, Joseph S Lam ${ }^{\mathrm{e}}$, Alexander W. Ensminger ${ }^{\mathrm{a}, \mathrm{b}}$, Karen L. Maxwell ${ }^{\mathrm{b}}$, and Alan R. Davidson $^{\mathrm{a}, \mathrm{b}} \#$

${ }^{\mathrm{a}}$ Department of Molecular Genetics, University of Toronto, Toronto, Ontario, Canada

${ }^{b}$ Department of Biochemistry, University of Toronto, Toronto, Ontario, Canada

${ }^{\mathrm{c} C h i l d ~ H e a l t h ~ R e s e a r c h ~ F o u n d a t i o n, ~ D h a k a, ~ B a n g l a d e s h ~}$

${ }^{\mathrm{d}}$ Department of Microbiology \& Immunology, Quantitative Biosciences Institute, University of California, San Francisco, San Francisco, California, United States.

${ }^{\mathrm{e}}$ Department of Molecular and Cellular Biology, University of Guelph, Guelph, Ontario, Canada \#Address correspondence to Alan R. Davidson, alan.davidson@utoronto.ca

Running Title: Characterization of F-type pyocins

Keywords: F-type pyocin, bacteriophage, Pseudomonas aeruginosa, R-type pyocin, bacteriocin, antibiotic alternative 


\section{ABSTRACT}

2 Most Pseudomonas aeruginosa strains produce bacteriocins derived from contractile or non-

3 contractile phage tails known as R-type and F-type pyocins, respectively. These bacteriocins

4 possess strain-specific bactericidal activity against $P$. aeruginosa and likely increase

5 evolutionary fitness through intraspecies competition. R-type pyocins have been studied

6 extensively and show promise as alternatives to antibiotics. Although they have similar

7 therapeutic potential, experimental studies on F-type pyocins are limited. Here, we provide a

8 bioinformatic and experimental investigation of F-type pyocins. We introduce a systematic

9 naming scheme for genes found in R- and F-type pyocin operons and identify 15 genes

10 invariably found in strains producing F-type pyocins. Five proteins encoded at the 3'-end of the

11 F-type pyocin cluster are divergent in sequence, and likely determine bactericidal specificity. We

12 use sequence similarities among these proteins to define 11 distinct F-type pyocin groups, five of

13 which had not been previously described. The five genes encoding the variable proteins associate

14 in two modules that have clearly re-assorted independently during the evolution of these operons.

15 These proteins are considerably more diverse than the specificity-determining tail fibers of R-

16 type pyocins, suggesting that F-type pyocins emerged earlier or have been subject to distinct

17 evolutionary pressures. Experimental studies on six F-type pyocin groups show that each

18 displays a distinct spectrum of bactericidal activity. This activity is strongly influenced by the

19 lipopolysaccharide O-antigen type, but other factors also play a role. F-type pyocins appear to

20 kill as efficiently as R-type pyocins. These studies set the stage for the development of F-type

21 pyocins as anti-bacterial therapeutics.

\section{IMPORTANCE}

23 Pseudomonas aeruginosa is an opportunistic pathogen that causes a broad spectrum of antibiotic

24 resistant infections with high mortality rates, particularly in immunocompromised individuals

25 and cystic fibrosis patients. Due to the increasing frequency of multidrug-resistant $P$. aeruginosa

26 infections, there is great interest in the development of alternative therapeutics. One alternative is

27 protein-based antimicrobials called bacteriocins, which are produced by one strain of bacteria to

28 kill other strains. In this study, we investigate F-type pyocins, bacteriocins naturally produced by

29 P. aeruginosa that resemble non-contractile phage tails. We show that they are potent killers of 
P. aeruginosa, and distinct pyocin groups display different killing specificities. We have

31 identified the probable specificity determinants of F-type pyocins, which opens up the potential

32 to engineer them to precisely target strains of pathogenic bacteria. The resemblance of F-type

33 pyocins to well characterized phage tails will greatly facilitate their development into effective

34 antibacterials.

\section{INTRODUCTION}

36 With increasing antibiotic resistance, there is a strong incentive to identify alternative anti-

37 bacterial therapeutics. To this end, interest in using phages or parts of phages to treat bacterial

38 infections has greatly increased in recent years (1), and phage treatments have proven effective

39 in clearing bacterial infections in humans (2-4). This success notwithstanding, there are potential

40 drawbacks to phage therapy, including the possibility that introduced phages may acquire and

41 transmit virulence or antibiotic resistance genes, and that negative outcomes may arise from

42 long-term phage reproduction within a patient. To circumvent these problems, the therapeutic

43 potential of phage tail-like bacteriocins, also referred to as tailocins, is also being explored.

44 Tailocin encoding operons, which are found in many diverse bacterial species, are likely derived

45 from prophages. The utility of tailocins as antibacterials has been amply demonstrated $(5,6)$.

46 Like phages, tailocins are highly specific for their target organism, but they possess additional

47 advantages. A single tailocin type can be engineered to kill a variety of bacterial species $(7,8)$,

48 and tailocins can be efficiently produced in easily cultured organisms, such as E. coli (9) or $B$.

49 subtilis (10). In this work, we provide a detailed investigation of a group of tailocins produced by

$50 \quad$ Pseudomonas aeruginosa that are related to non-contractile phage tails.

51 The tailocins of $P$. aeruginosa, discovered many decades ago (11), fall into two types known as

$52 \quad$ F-type pyocins and R-type pyocins. All P. aeruginosa strains possess a gene cluster located

53 between the $\operatorname{trp} E$ and $\operatorname{trp} G$ genes encoding F-type, R-type or both types of pyocins. R-type

54 pyocins, which are related to contractile-tailed phages, such as E. coli phage P2 (12), have been

55 extensively studied. These entities are produced by different strains of $P$. aeruginos $a$ and have

56 the ability to kill other strains of the same species. R-type pyocins bind specifically to target

57 strains, and then puncture their inner membrane, leading to rapid cell death (13). Derivatives of

58 R-type pyocins with engineered tail fibres are able to kill other species of bacteria, such as $E$. 
coli and Yersinia pestis, and these engineered variants have shown efficacy in preventing and/or

60 ameliorating infection in animal models $(6,9,14,15)$. F-type pyocins, which are related to non-

61 contractile tailed phages, such as E. coli phage lambda (12), have been studied much less than

62 the R-type. Although encoded in more than half of P. aeruginosa strains (16), no experimental

63 work has been published on F-type pyocins since 1981 (17). The activities of F-type pyocins

64 produced by five different strains have been described in the literature (17-20), each of which

65 killed distinct sets of $P$. aeruginosa strains. However, the sequences of the operons encoding

66 only two of these are known. Based on genome sequencing data, four further groups have been

67 defined (16), but neither the production nor activity of these groups was assessed. The

68 mechanism of action and killing specificity determinants of F-type pyocins have not been

69 defined.

70 Given the potential importance of tailocins in treating bacterial infections and the relative dearth

71 of information pertaining to F-type pyocins, we undertook a comprehensive investigation of F-

72 type pyocins encoded in a large number of $P$. aeruginosa strains. The goals of this study were to

73 bioinformatically characterize F-type pyocin operons and correlate sequence diversity with the

74 killing spectra of defined F-type pyocin groups. Through this process, we have identified 11

75 distinct groups of F-type pyocins, and their likely specificity determinants. We conclude that

76 these F-type pyocins have the potential to be engineered as highly effective anti-bacterial

77 therapeutics.

\section{RESULTS}

79 Selection of $\boldsymbol{P}$. aeruginosa strains for this study

80 To gain an understanding of the diversity of R-type and F-type pyocins produced by $P$.

81 aeruginosa, we produced lysates of diverse strains selected from our collection (21) by treating

82 cultures with mitomycin $\mathrm{C}$, which induces pyocin production and cell lysis (22). Each of these

83 lysates was examined by transmission electron microscopy (TEM), and those displaying

84 abundant levels of R- and/or F-type pyocins were further analyzed (Fig. 1). Ultimately, a set of

8530 strains was chosen that produced only F-type $(n=8)$, only R-type $(n=9)$ or both R- and F-

86 type $(\mathrm{n}=13)$ pyocins. This set contained clinical and environmental strains from seven different

87 countries, collected over a few decades (Supplementary Table 1). Twenty-eight of the 30 strains 
were sequenced, assembled and annotated in this study (annotated genomes of strains PAO1 and PA14 were obtained from the Pseudomonas Genome Database (23)).

91 The R- and F-type pyocin gene clusters are invariably found between the $\operatorname{trp} E$ and $\operatorname{trp} G$ genes in

$92 P$. aeruginosa (12). To locate these gene clusters in each genome that was sequenced in this

93 study, the regions between gene $\operatorname{trp} E$ and $\operatorname{trp} G$ were extracted and analyzed (Fig $2 \mathrm{a}$ ). Each of the

9430 sequenced genomes was found to encode an F-type- or R-type pyocin, or both, corresponding

95 with the observed production of pyocin particles observed by electron microscopy. In total, 23 R-

96 type and 21 F-type pyocin gene clusters were present in our analyses. The gene content of the

97 pyocin clusters was constant across all the strains. We observed eight genes, designated pyoRF1

98 to $p y o R F 8$, which were found in all clusters, 15 genes specific to R-type pyocins (pyoR1 to

99 pyoR15), and 15 genes specific to F-type pyocins (pyoFl to pyoF15) (Fig. 2a, Table 1). The

100 pyoRF1 and pyoRF2 genes encode the PrtN activator and PrtR repressor, respectively. These

101 proteins regulate expression of the cluster in response to DNA damage as previously described

102 (22). The pyoRF3 and pyoRF4 gene products are uncharacterized, but their predicted functions

103 suggest a role in regulating expression of the gene cluster. Analysis by HHpred indicates that the

104 pyoRF3 gene encodes a putative zinc-binding transcription factor and pyoRF4 encodes a putative

105 transcription anti-terminator protein similar to gpQ of phage lambda (24). Homologs of PyoRF3

106 are found in more than 100 phage and prophage genomes, while homologs of PyoRF4 are found

107 in a much smaller number of phages and prophages. The pyoRF5 to pyoRF8 genes encode a

108 complete set of phage-like lysis genes, including a peptidoglycan hydrolase, holin, and Rz and

109 Rz1-like spannins (25). In operons encoding only F-type pyocins all eight pyoRF genes precede

110 the genes encoding the F-type pyocin specific genes. In clusters encoding just R-type pyocins, or

111 those encoding both R- and F-type pyocins, the R-type pyocin specific genes are inserted within

112 the lysis gene cluster between pyoRF5 and pyoRF6 (Fig. 2a).

113 Within the 22 R-type pyocin clusters in the genomes studied here, 13 of the 15 encoded proteins

114 are highly conserved among the clusters, with at least 97\% sequence identity between each gene

115 product, as has been previously documented (15). The two proteins that vary significantly are

116 PyoR6 and PyoR7, which encode the tail fiber and tail fiber chaperone, respectively. The tail

117 fiber determines the specificity of R-type pyocins and the chaperone is specific to its cognate 
118 fiber. We compared the fiber and chaperone proteins of each of our sequenced clusters to those

119 of the characterized R-type pyocin types (15). Fiber sequences of the R2-, R3- and R4-types are

120 very similar to each other ( $>98 \%$ identical). Hence, we considered groups R2, R3 and R4 as one

121 group and called it group R2, as was done in a previous study (26). For the R-type pyocin

122 clusters sequenced here, ten belonged to the R1 group, ten to the R2 group, and two to the R5

123 group. As R-type pyocins have been well characterized in previous studies $(7,8,15)$, we focused

124 the present investigation on the F-type pyocins.

\section{Conserved proteins encoded in the F-type pyocin cluster}

126 The pyoF2 to pyoF10 genes encode confidently annotated functions required for formation of the

127 F-type pyocin tube and tip (Table 1). The protein products of each of these genes are clearly

128 homologous to phage tail proteins (27), and these proteins are very similar ( $95 \%$ pairwise

129 sequence identity) among the 21 F-type pyocin gene clusters that we have analyzed. Although

130 the F-type pyocin genes are arranged in an order that is syntenic with the genome of the well

131 characterized E. coli phage lambda (12), only the tail tip and central fiber proteins (PyoF6 to

132 PyoF10) of this phage share significant sequence identity with F-type pyocin proteins (31 to $38 \%$

133 sequence identity). The phage tail region with the greatest similarity to the F-type pyocin cluster

134 across the tube and tail tip region is that from E. coli phage HK022. (Table 1). The HK022

135 proteins share $43 \%$ sequence identity, on average, to those of the F-type pyocin (Table 1). No

136 prophage tail region was more closely related to the F-type pyocin cluster than phage HK022

137 across the whole cluster, though some $P$. aeruginosa prophages were more closely related to the

1383 '-end of the cluster where genes encoding the tail tip proteins are located.

139 An unusual feature of F-type pyocin regions as compared to phage tails is the lack of any protein

140 with detectable similarity to a tail terminator. This protein is essential for phage tails because it is

141 required to join the tail to the head (28). The tail terminator also prevents uncontrolled

142 polymerization of the tails of some (28), but not all phages (29). Since F-type pyocins are not

143 joined to a head, the tail terminator appears to be dispensable. The pyoF1 gene lies in the

144 genomic position expected for a tail terminator gene. However, the 95 amino acid protein

145 encoded by this gene bears no detectable sequence similarity to tail terminators, has no homologs

146 outside of $P$. aeruginosa F-type pyocin clusters, and stop codons are observed in this ORF in 
147 several strains. Thus, we conclude that this is not a functioning protein as was also concluded in

148 a previous publication (12).

\section{F-type pyocins can be grouped based on proteins encoded at the 3'- end of the cluster}

151 The host range specificity of phages is determined by proteins located at the tail tip, which are

152 typically encoded by genes at the 3'-end of tail-encoding regions (27). The analogous proteins in

153 the F-type pyocin are encoded by genes pyoF10-pyoF15. Non-contractile tails resembling F-type

154 pyocins possess a long ( $>700$ residues) central fibre protein that projects directly below the tail

155 tip. In phage lambda, the region within the last 250 residues of the central fiber mediates host

156 cell specificity and surface binding $(30,31)$. The homologous protein in F-type pyocins is

157 encoded by pyoF10. We observed that the first 1160 residues of the PyoF10 proteins are highly

158 conserved among F-type pyocins (>93\% sequence identity), but the last 60 residues vary greatly,

159 with pairwise identities in this region often ranging below 35\% (Fig. S1). This sequence

160 variability is consistent with a role for the C-terminus of PyoF10 in mediating host specificity.

161 In addition to the last 60 residues of PyoF10, the other five proteins encoded at the 3'-end of the

162 F-type pyocin cluster, PyoF11 to PyoF15, were found to vary considerably in sequence between

163 different F-type pyocin clusters. Based on pairwise comparison of homologous proteins encoded

164 in this region of the clusters (Fig. S1), the F-type pyocin regions found in different genomes were

165 divided into six groups, F1, F2, F4, F5, F6 and F7 (Fig. 2b). Regions were placed into the same

166 group if each of their corresponding homologous proteins shared at least $90 \%$ sequence identity

167 with all others in the group. The nomenclature used here extends from previous work where

168 groups F1 to F3 were established based on differences in host killing specificity (17). We do not

169 know if any of the groups identified here match group F3 because no examples from this group

170 have been sequenced. The groups that we called F4 and F6 have not been previously recognized,

171 while group F5 and F7 were previously described in P. aeruginosa strains PA14 and DK2,

172 respectively (16). The two most frequently occurring groups are F2 (11 members) and F7 (4

173 members). The F1, F5 and F6 groups were encoded only in pyocin clusters that also encoded R-

174 type pyocins, while F4, F7, and F2 group clusters were found in the absence of R-type clusters

175 except in two instances (both F2 group). Further bioinformatic comparisons described below 
compare representative protein sequences from each of the six F-type pyocin groups that we identified here.

\section{PyoF11 and PyoF12 are newly recognized conserved proteins}

PyoF11 and PyoF12 are proteins of unknown function that are encoded in every F-type pyocin region. These are the most diverse proteins in the F-type pyocin clusters, often displaying pairwise sequence identities of less than 25\% (Fig. S1). Despite their diversity, the homologs of these proteins from the six groups could be convincingly aligned (Fig. S2a,b). We used HMMer (32) to create Hidden Markov Model (HMM) profiles from the PyoF11 and PyoF12 alignments. Searching with these HMMs, we identified more than 50 occurrences each of pyoF11 and pyoF 12 gene homologs in diverse phages and prophages. These genes often occur together and invariably lie immediately 3 ' to the central fiber gene (homolog of pyoF 10). In some phage genomes, the pyoF11 and pyoF 12 genes are very likely the last genes in the tail operon as they are followed immediately by lysis genes (e.g. Burkholderia phage Bcep176 and Xanthomonas phage CP1). These observations suggest that PyoF11 and PyoF12 function in conjunction with the central fiber protein, possibly binding to it or acting as chaperones to aid in folding of the fiber. PyoF11 and PyoF12 had not been previously recognized as conserved proteins in the Ftype pyocin cluster because these ORFs are not annotated as proteins in most $P$. aeruginosa genomes. This is likely a result of the lack of annotation of these genes in the PA14 genome, which is commonly used as the reference genome for genome assembly and annotation. The functions of PyoF11 and PyoF12 homologs in phages have never been investigated.

\section{PyoF13, PyoF14, and PyoF15 are likely involved in host specificity}

In addition to the central fiber, most non-contractile tailed phages possess genes downstream of the central fiber gene that also encode cell surface receptor binding proteins These are known as "side fibers" in E. coli phage lambda (33). The PyoF13 proteins, which share a common genomic position with the lambda side fibers, are likely involved in determining host range specificity, functioning as receptor binding proteins. A striking feature of the Pyo13 homologs is that their $\mathrm{N}$-termini (the first 140 residues) are very similar among the F-type pyocin groups with pairwise sequence identities ranging from $55 \%$ to $90 \%$ while the pairwise identities in their C-terminal regions generally range between $20 \%$ and $35 \%$ (Fig. S1, S3). We surmise that the more 
conserved N-terminus of PyoF13 mediates binding of this putative receptor binding protein to the F-pyocin tail tip, while the variable C-terminus mediates cell surface binding. The fibers

207 from different groups of R-type pyocins, which have been shown to determine bactericidal specificity (15), display the same type of conservation pattern with N-terminal regions (first 450 residues) displaying greater than $95 \%$ pairwise sequence identity and C-terminal regions (last 250 residues) displaying pairwise sequence identities between 50 and 70\% (Fig. S4). In contrast

211 to the F-type pyocins, there are only three distinct groups of R-type pyocins, as defined by fiber

212 sequences, and there is much less variability.

213 Homologs of PyoF13, which share sequence similarity with its N-terminal region, are found in

214 diverse phages and prophages and are located in similar genomic positions as pyoF13,

215 downstream from the central fiber gene. Genes encoding homologs of PyoF14 ( $\sim 100$ residues)

216 and PyoF15 ( 75 residues) are also found in many phages and prophages, and they are invariably

217 located downstream of pyoF13 homologs or genes encoding other putative phage receptor-

218 binding proteins. The sequences of PyoF14 and PyoF15 are variable, mirroring the sequence

219 variation seen in the C-terminal regions of PyoF13 (Fig. S1). We expect that PyoF14 and

220 PyoF15 are involved in host range specificity through interactions with PyoF13, or possibly by

221 acting as chaperones for the assembly of PyoF13 as is required for phage-encoded receptor

222 binding proteins (34).

223 Two F-type pyocin groups deviate from the others in the pyoF13 to pyoF15 region. The F2

224 group has a complete duplication of this region so that it possesses two copies of each gene. The

225 proteins encoded by the first copy, PyoF $13_{1}$ to PyoF15, are distinct in sequence compared to the

226 homologs in other groups (Fig. S1, Fig S3). Conversely, PyoF13 2 to PyoF $15_{2}$ are very similar (>

$22790 \%$ identical) to homologs found in groups F4 and F5. In contrast to all other groups, group F7

228 lacks a pyoF14 gene. Consistent with this absence, its PyoF13 homolog displays a C-terminal

229 region that has no detectable sequence similarity to the other groups.

\section{Characterization of F-type pyocin bactericidal specificity}

231 To determine if our bioinformatic groupings of the F-type pyocin clusters correlate with

232 bactericidal specificity, we examined the killing profiles of lysates produced from the 30 strains

233 following induction by mitomycin C. Serial dilutions of lysates of each of the 30 strains were

234 spotted onto lawns of the same 30 strains to produce an all-against-all matrix. Bactericidal 
activities were detected as zones of clearing on the bacterial lawn. Analysis of these data was

236 complicated because $P$. aeruginosa produces other bactericidal entities in addition to R-type and

237 F-type pyocins, including S-type pyocins (11) and bacteriophages. Since the presence of any of

238 these can produce zones of clearing, further analyses were necessary to delineate the type of

239 activity present. Testing serial dilutions of lysates allowed us to distinguish clearings produced

240 by phages from those produced by pyocins (Fig. 3a). Due to their replicative nature, clearings

241 resulting from phage lysates resolved into individual plaques upon dilution, while the clearings

242 resulting from pyocins gradually disappeared without the appearance of individual plaques (Fig.

243 3a). Lysates were also spotted onto bacterial lawns containing proteinase $\mathrm{K}$, which eliminated

244 clearings caused by protease sensitive S-type pyocins (Fig. 3a) (11). By analyzing the activities

245 of the 30 lysates on 30 strains in this manner, we detected more than 450 bactericidal

246 combinations and found that greater than 90\% were due to R- or F-type pyocins (Fig. S5).

247 All groups of R-type and F-type pyocins identified displayed bactericidal activities against

248 multiple strains. Notably, the killing spectra of lysates were invariably the same if they contained

249 pyocins of the same R- or F-type group (Fig. S5). For example, lysates of four different strains

250 encoding F7 pyocins all displayed bactericidal activity against the same 11 strains (note that in a

251 single case the F-type pyocin activity was occluded by the presence of phage activity as denoted

252 by an orange color, Fig S5). These results demonstrate that our classification of pyocins based on

253 sequence analysis is predictive of biological activity. In Fig. 3b, a small subset of the bactericidal

254 data are shown to emphasize the differences in the killing spectra of the F-type pyocin groups.

255 No two groups kill exactly the same set of bacterial strains; however, considerable overlap exists

256 between some groups like F4 and F5. We also noted that no strain was susceptible to an R- or F-

257 type pyocin that was encoded in its own genome, which is consistent with previous observations

258 that strains are resistant to their own pyocins (35). Since the F1 and F6 groups were encoded

259 only in strains that also encoded R1 pyocins, the killing caused only by the F-type pyocins could

260 not be discerned. However, comparison with results obtained using a strain encoding only an R1

261 pyocin revealed that strain S25 is susceptible to F1 pyocin as it was killed by a lysate containing

262 F1 and R1 pyocins, but not by a lysate containing only R1 pyocin (Fig. 3b). By the same logic,

263 strain S30 was found to be killed by F6 pyocin. The F5 group was found only in strains that also

264 encode an R-type pyocin. To assess the activity of this group we took advantage of a transposon 
insertion mutant of an essential R-type pyocin gene in PA14 (36) to detect the activity of the F5 pyocin alone (Fig. 3b).

\section{F-type and R-type pyocins display similar levels of bactericidal}

\section{activity}

269 It was previously reported that one R-type pyocin particle is sufficient to kill a single cell, while 270 up to 280 F-type pyocin particles are required to kill the same cell (19). This implies that an F-

271 type pyocin containing lysate would have considerably less killing activity than an R-pyocin

272 containing lysate. However, we observed many cases where lysates of F-type pyocins displayed

273 levels of killing activity as high R-type pyocin lysates. Although R-and F-type pyocin lysates

274 may contain different numbers of particles, we do not expect these numbers to deviate greatly as

275 all pyocin operons utilize the same transcriptional regulatory region. Most convincingly, we

276 tested the bactericidal activity of lysates of two PA14 mutants, one of which carried a transposon

277 insertion in an essential R-type pyocin gene (pyoR6) and one of which carried a similar insertion

278 in an essential F-type pyocin gene (pyoF10). It can be seen that the bactericidal activity of these

279 two lysates was the same, indicating that F-type pyocins and R-type pyocins are equally lethal to

280 a susceptible host (Fig. 3c). We observed that the same F-type pyocin lysate may display

281 different levels of activity on different strains. For example, lysates of F7 group pyocins

282 displayed greater than 10-fold greater bactericidal activity on strain PAO1 as on strain S14 (Fig.

283 3d). The previously observed low activity of F-type pyocins was likely caused by use of a non-

284 optimal indicator strain. Overall, our data indicate that F-type pyocins have the potential to kill

285 bacterial cells as efficiently as R-type pyocins.

\section{The genes downstream of pyoF10 are required for bactericidal activity}

287 Although homologs of the proteins encoded at the 3'-end of the F-type pyocin cluster (PyoF11 to

288 PyoF15) are encoded in phages and prophages, the roles of these proteins have never been

289 investigated. To determine whether these proteins are essential for bactericidal activity, we tested

290 the activity of F-type pyocin mutants in strain PA14 (group F5). We tested transposon insertion

291 mutations in pyoF14, and pyoF15 from the PA14 non-redundant transposon mutant library (36).

292 We constructed in-frame deletion mutations in pyoF11 and pyoF12 and a nonsense mutation in

293 pyoF13 (Supplementary Materials and Methods). Mutations in each of these genes completely 
abrogated bactericidal activity, indicating that their protein products play essential roles in the production of functional F-type pyocin particles (Table 2). To ensure that the loss of activity resulting from these mutations was the result of abrogation of only the gene in which the mutation was located, each gene was cloned into a plasmid expression vector (Supplementary Materials and Methods) and we determined whether mutations could be complemented by the plasmid expressed genes. The pyoF 12 mutant could be complemented by a plasmid expressing only pyoF12 (Table 2). However, complementation of the pyoF11 mutant required plasmidbased expression of both pyoF 11 and pyoF 12. A plasmid expressing only pyoF 12 did not complement the pyoF11 mutant. We conclude that both pyoF11 and pyoF12 are essential for bactericidal activity, and that the $p y o F 11$ in-frame deletion mutation also causes loss of pyoF 12 activity, possibly through a polarity effect. Through a similar series of plasmid based complementation experiments, we determined that pyoF13, pyoF14, and pyoF15 are also essential for bactericidal activity, and that polarity effects are also manifested in this group of genes (Table 2). For example, while the pyoF 15 mutation could be complemented by expression of pyoF15 alone, complementation of the pyoF14 mutation required expression of both pyoF14 and pyoF 15 .

\section{Serotype correlates with F-type pyocin killing spectra}

311 The outer membrane lipopolysaccharide (LPS) of $P$. aeruginosa is composed of three domains:

312 lipid A, core oligosaccharide and a long-chain polysaccharide O-antigen (37). Most $P$.

313 aeruginosa strains produce two distinct forms of O-antigen; a homopolymer of D-rhamnose

314 known as the common polysaccharide antigen, and a heteropolymer repeat of three to five

315 distinct sugars known as the O-specific antigen (OSA), which forms the basis of P. aeruginosa

316 serotyping. Previous studies showed that the OSA acts a receptor for some R-type pyocins, while

317 it blocks killing by other R-type pyocins (26). To investigate the effect of the OSA on the

318 activity of F-type pyocins, we experimentally determined the serotypes of the 30 strains used in

319 this study by a slide agglutination assay. We observed a correlation between the serotype of a

320 strain and its F-type pyocin susceptibility profile (Fig. 4a). For example, all three strains of $\mathrm{O} 2$

321 serotype were resistant to all F-type pyocins, while the four O5 strains were killed only by F7

322 pyocins. Among the eight O6 serotype strains, the F2 pyocin killed all, but the F4, F5, and F7

323 pyocins were unable to kill some of these strains (Fig. 4a). The resistance of O6 strains S12 and 
324 S27 to the activity of the F4 pyocin is expected as these strains encode an F4 pyocin. However, it 325 is not clear why the F7 pyocin fails to kill O6 strains S12, S27, and S5, or why strain S12, alone 326 among O6 strains, is resistant to F5 pyocin. Similarly, the F1 pyocin kills strain S25 but no other 327 O6 strains, and the F6 pyocin kills strain S6 but no other O13/O14 strains. These data show that 328 factors independent of OSA and pyocin type encoded within a strain contribute to F-type pyocin 329 susceptibility.

330 To directly assess the role of OSA in F-type pyocin activity, we tested $\Delta w b p M$ mutant strains, 331 which lack OSA in strains PAO1 and PA14 (Fig 4b). The F7-type pyocin is active against PAO1, 332 but was unable to kill the PAO1 $\triangle w b p M$ mutant, suggesting that this pyocin uses the OSA as a 333 receptor. By contrast, the $\triangle w b p M$ mutants of PAO1 and PA14 became susceptible to the F4

334 group, though the wild-type strains were not. In this case, the OSA appears to block the pyocin

335 from contacting its receptor. The F2 and F5 groups, which are unable to kill PAO1 or PA14,

336 were also not able to kill the mutants lacking OSA. Strains producing the F1 and F6 group

337 pyocins were unable to kill PAO1 with or without OSA, but PA14 $1 w b p M$ did become

338 susceptible to killing. However, this effect may have been due to the R1 pyocins produced by

339 these strains. From these experiments with strains lacking OSA, it is clear that the presence of

340 OSA affects the bactericidal activity of the F4 and F7 groups while the data are inconclusive for 341 the other groups.

\section{Discovery of new groups of F-type pyocins}

343 To determine if this collection of F-type pyocin described above encompassed the full diversity

344 of F-type pyocins found across the P. aeruginosa species, we performed BLAST searches

345 against all P. aeruginosa genomes in the NCBI database using a PyoF13 sequence as the query

346 with the goal of identifying homologs with distinct sequences (i.e. share less than $90 \%$ sequence

347 identity with those in our established F-type pyocin groups). PyoF13 was chosen for these

348 searches because it is highly conserved among the F-type pyocin operons in its N-terminal

349 region, yet its $\mathrm{C}$-terminal region varies depending on the pyocin group. We discovered three

350 PyoF13 homolog families encoded in F-type pyocin operons that shared less than 70\% sequence

351 identity to any other PyoF13 group. F-type pyocins encoding these newly identified PyoF13

352 varieties were defined as groups F8, F9, and F10. The F9 group is identical to a previously

353 identified group designated as the PA7 group (16). Another identified group, called F11, 
354 possessed PyoF13, PyoF14, and PyoF15 homologs that are greater than 95\% identical to group

355 F2 2, F4 and F5, but the PyoF10, PyoF11, and PyoF12 were unique. Finally, group F12 combined

356 PyoF10 to PyoF12 homologs that were 99\% identical to group F11 with PyoF13 to PyoF15

357 homologs that were greater than 95\% identical to group F10 (Fig. 5, S1). Group F12 was

358 previously identified in P. aeruginosa strain M18 (16). The sequences of proteins PyoF10 to

359 PyoF15 for all eleven F-type pyocin groups can be found in Appendix 1 (Supplementary

360 Material).

361 Pairwise sequence comparisons among all the F-type pyocin groups that we have identified 362 strongly supports the existence of two distinct specificity modules in F-type pyocins (Fig. S1).

363 Whenever the C-terminal regions of PyoF10 proteins in two groups are highly similar $(>90 \%$

364 identity), then the PyoF11 and PyoF12 proteins are also highly similar. Similarly, when two

365 groups have PyoF13 proteins with highly similar C-terminal regions, then the PyoF14 and

366 PyoF15 proteins are also highly similar. Therefore, we have designated regions encoding the C-

367 terminus of PyoF10, PyoF11, and PyoF12 as Specificity Module 1 and regions encoding

368 PyoF13, PyoF14, and PyoF15 as Specificity Module 2. Among our full set of pyocin groups, we

369 observed three instances where the same Specificity Module 1 region assorted with different

370 Specificity Module 2 regions (Fig. 5). We also observed three cases where identical Specificity

371 Module 2 regions assorted with different Specificity Module 1 regions. These data indicate that

372 recombination events have occurred between different F-type pyocin operons.

\section{DISCUSSION}

374 This study provides a comprehensive analysis of F-type pyocin operons present in P. aeruginosa

375 strains. We have defined the conserved genes in these operons and introduced a systematic

376 naming system for them. The initial $21 \mathrm{~F}$-type clusters examined in strains from our collection

377 were categorized into six different groups, two of which were previously known (F1 and F2) and

378 four of which were named in this study (groups F4 to F7). The killing specificity of each of these

379 groups was shown to be distinct. An additional five F-type pyocin groups were discovered

380 bioinformatically, but the killing specificity of these groups remains to be tested. Importantly, we

381 identified two highly diverse F-type pyocin genes, pyoF11 and pyoF12, which are not annotated

382 as genes in many $P$. aeruginosa strains, yet are essential for bactericidal activity. The sequence 
diversity in these two genes contributes to defining the F-type pyocin groups. An important

384 finding is that there are no genes in the F-type pyocin operons that are not also found in the genomes of phages or prophages. Thus, the ability of these pyocins to efficiently kill bacteria while isolated phage tails do not must be due to sequence modifications within their phagederived proteins, not to the presence of unique toxin-encoding genes (unless such genes are encoded elsewhere in the $P$. aeruginosa genome). Fully active R-type pyocins have been produced heterologously in $E$. coli from a plasmid vector including only genes from the R-type pyocin operon, indicating that their toxicity does not rely on genes outside of this region (9).

Analysis of the F-type pyocin operons clearly indicates the genes that are involved in killing specificity. The proteins encoded by the $\mathrm{pyoF}^{2}$ to $\mathrm{pyoF}^{9}$ genes are highly similar in all the F-

393 type pyocin groups. Divergence among the groups begins with the last 60 residues of PyoF10 and extends through PyoF15. We defined the F-type pyocin groups according to sequence identity among these proteins (Fig. 5). Since F-type pyocins within the same group invariably displayed the same killing spectra (Fig. S5), we conclude that some or all of the pyoF10 to pyoF 15 region determines killing specificity. By examining the patterns of recombination among the specificity genes, we defined two specificity modules: Module 1 (pyoF10 to pyoF12) and Module 2 (pyoF13 to pyoF15). The occurrence of highly similar Module 1 regions with distinct Module 2 regions and vice versa in different F-type pyocin groups indicates that the two modules act independently of one another (Fig. 5). This conclusion is supported by the appearance of pyoF11 and pyoF 12 homologs without adjacent pyoF13-pyoF15 homologs and vice versa in phages and prophages. Since these families of proteins have not been characterized, defining their roles in host specificity will be an important goal for further study.

The strong sequence similarity between most of the F-type pyocin genes implies that all the groups are descended from a common ancestor that likely arose from a defective prophage. However, it is also clear that some type of horizontal gene transfer mechanism has been responsible for the evolution of the specificity regions, which are comprised of different 410 carrying genes that are similar to these F-type pyocin genes occasionally recombining with the

411 homologous F-type pyocin genes. With respect to the evolution of the F-and R-type pyocins as a 412 whole, it is relevant that the F-type display considerably more divergence among their 413 specificity-determining genes as compared to the R-type (Fig. S1, S4). This suggests that the F- 
414 type pyocins may have arisen first and, thus, have had more time to diverge. Also supporting the

415 possibility that the F-type pyocin operon appeared first is that the R-type pyocin genes are

416 inserted in the middle of the lysis genes, which comprise an intact lysis cassette in strains

417 possessing only an F-type operon.

418 The bacterial cell surface receptors of F-type pyocins were previously unknown. Our

419 examination of the activity of the different F-type pyocin groups on 12 different serotypes of $P$.

420 aeruginosa revealed a clear correlation between bactericidal activity and O-antigen serotypes

421 (Fig. 4a). Further supporting a role for the OSA in the activity of at least some F-type pyocins is

422 the fact that activity of the F7 group required the presence of OSA, while group F4 activity was

423 blocked by OSA (Fig. 5b). While the OSA serotype clearly influences F-type pyocin host

424 recognition, this is not the only determining factor. For example, the F7 pyocin is able to kill

425 some but not all strains with the O6 serotype. In addition, F-type pyocins of a given type were

426 consistently unable to kill strains encoding the same type of F-type pyocin, regardless of

427 serotype. The mechanism of this self-immunity is not known. Many bacteriocins, such as S-type

428 pyocins and colicins, are encoded with specific immunity proteins (11). However, there is no

429 obvious immunity protein candidate encoded within F-type pyocin clusters as each gene is

430 homologous to phage tail proteins. It is possible that no specific immunity proteins exist for R-

431 type or F-type pyocins. Rather, strains may have evolved to resist their resident R- and F-type

432 pyocins by altering their cell surface in subtle ways undetectable by the antibodies used in

433 serotyping.

434 Overall, our study shows that F-type pyocins are produced by a large number of $P$. aeruginosa

435 strains, they all possess antimicrobial properties, and they are promising candidates to study for

436 the development of new therapeutics. Our identification of the specificity determinants of F-type

437 pyocins points the way toward precisely engineering their killing as has been done with the

438 contractile R-type pyocins and non-contractile tailocins of Listeria $(7,8,10)$. 


\section{MATERIALS AND METHODS}

\section{Whole genome sequencing}

442 Genomic DNA was isolated using a genomic DNA extraction kit (Bio Basic Inc). Next-

443 generation whole genome sequencing was performed by the Donnelly Sequencing Center,

444 University of Toronto, using Illumina HiSeq2500. De novo assembly of reads into contigs was

445 performed using Velvet version 2.2.5 (38). Genes $\operatorname{trp} E$ and $\operatorname{trp} G$ were located and the region

446 between these genes was analyzed using Geneious (39).

\section{Bioinformatic analysis}

448 Most of the bioinformatic analyses, including BLAST (40) searches and genome synteny

449 analyses were carried out on a custom database comprised of 755 tailed phage genomes and

4502,119 bacterial genomes downloaded from the National Center for Biotechnology Information

451 (NCBI) Refseq database in April 2013. This database contains diverse phage and bacterial

452 species, but was small enough to allow manual analysis of the protein sequences and the

453 genomic context of genes encoding proteins related to pyocin proteins. This work was aided by a

454 synteny viewing and phage gene annotation toolkit developed in our laboratory, which will be

455 described in detail elsewhere. Sequence alignment analysis was performed in Jalview (41). To

456 identify protein sequences similar to less frequently occurring proteins found in the pyocin

457 cluster (e.g. PyoR1, PyoF11, and PyoF12), alignments were constructed of the pyocin proteins.

458 HMMER3 (32) was then used to create Hidden Markov Models (HMMs). These HMMs were

459 used to detect proteins similar to a given pyocin protein. The genome context of genes encoding

460 these similar proteins within phage genomes was assessed to support a conclusion that the pyocin

461 protein possesses the same function as the phage protein. BLAST searches to identify new

462 groups of F-type pyocins were carried out against all $P$. aeruginosa genomes available at NCBI

463 in April, 2018.

464 HHpred searches were carried out using the online server

465 (https://toolkit.tuebingen.mpg.de/hhpred) (42). HMM-based searches were carried out using

466 HMMer (32) and analyzed by searching the Pfam (43) and TIGRfam (www.jcvi.org/cgi-

467 bin/tigrfams/index.cgi) databases. 


\section{Transmission electron microscopy}

469 A continuous carbon film coated EM grid was made hydrophilic by glow discharge. $5 \mu$ of sample was applied to the surface of the grid and left for absorption for 2 minutes. Excess sample was blotted away using the corner of a filter paper. The grid was washed three times with water and stained with 2\% (w/v) uranyl acetate. Grids were examined with a Hitachi H-7000 microscope.

\section{Assays of pyocin and phage bactericidal activity}

475 To generate lysates containing pyocins and/or phages, $5 \mathrm{ml}$ cultures started from overnights were 476 grown in $\mathrm{LB}$ at $30^{\circ} \mathrm{C}$ until the cells reached an $\mathrm{OD}_{600}$ of 0.4 . Mitomycin $\mathrm{C}$, was then added to a 477 final concentration of $2 \mu \mathrm{g} / \mathrm{ml}$ and shaking at $30^{\circ} \mathrm{C}$ was resumed for $3 \mathrm{~h}$ or until cell lysis 478 occurred. Chloroform was added to all induced cultures (1-2 drops/ml) to ensure maximum 479 bacterial lysis. In experiments testing complementation from plasmids, $0.2 \%$ arabinose was 480 added to cells after $1 \mathrm{~h}$ of growth at $30{ }^{\circ} \mathrm{C}$ to induce the expression of proteins from the plasmid 481 prior to addition to mitomycin $\mathrm{C}$ to induce F-type pyocin induction from the genome. After lysis, 482 cultures were incubated at room temperature with DNase $(10 \mu \mathrm{g} / \mathrm{ml})$ for $30 \mathrm{~min}$ prior to centrifugation at $10000 \mathrm{rpm}$ for $10 \mathrm{~min}$. For activity assays, $2 \mu 1$ volumes of dilutions of these

484 lysates were spotted onto lawns of $P$. aeruginosa strains. Lawns of strains to be tested were made 485 by adding $150 \mu \mathrm{l}$ of overnight culture to $3 \mathrm{ml}$ of molten $0.7 \%$ top agar, which was immediately 486 poured onto an LB agar plate and allowed to harden. To distinguish S-type pyocin activity, 487 duplicate lawns were poured containing proteinase $\mathrm{K}(100 \mu \mathrm{g} / \mathrm{ml})$. At least three biological 488 replicates were performed for each strain and lysate combination. A lysate was scored as positive 489 if it displayed a strong zone of cell growth inhibition in every assay. There was a range of 490 activity in positive lysates with some displaying moderate zones of growth inhibition even when 491 diluted $10^{2}$-fold and others displaying strong activity only when undiluted. Lysates scored as 492 negative displayed very weak or no zones of growth inhibition in all replicate assays.

\section{Serotyping of $\boldsymbol{P}$. aeruginosa strains}

494 Strains were serotyped using the slide agglutination method using commercial antisera (MAST

495 Diagnostics) against all 20 P. aeruginosa serotypes recognized by the International Antigenic 496 Typing Scheme (37). 


\section{ACKNOWLEDGEMENTS}

499 This work was supported by operating grants from the Canadian Institutes of Health Research

500 (CIHR) to A.R.D. (XNE-86943 and FDN-15427), K.L.M (MOP-136845), and A.W.E. (PHT-

501 148819). S.S. was supported by a CIHR Doctoral Scholarship.

\section{REFERENCES}

1. Vandenheuvel D, Lavigne R, Brussow H. 2015. Bacteriophage therapy: advances in formulation strategies and human clinical trials. Annu Rev Virol 2:599-618.

2. Chan BK, Turner PE, Kim S, Mojibian HR, Elefteriades JA, Narayan D. 2018. Phage treatment of an aortic graft infected with Pseudomonas aeruginosa. Evol Med Public Health 2018:60-66.

3. Dedrick RM, Guerrero-Bustamante CA, Garlena RA, Russell DA, Ford K, Harris K, Gilmour KC, Soothill J, Jacobs-Sera D, Schooley RT, Hatfull GF, Spencer H. 2019. Engineered bacteriophages for treatment of a patient with a disseminated drug-resistant Mycobacterium abscessus. Nat Med 25:730-733.

4. Schooley RT, Biswas B, Gill JJ, Hernandez-Morales A, Lancaster J, Lessor L, Barr JJ, Reed SL, Rohwer F, Benler S, Segall AM, Taplitz R, Smith DM, Kerr K, Kumaraswamy M, Nizet V, Lin L, McCauley MD, Strathdee SA, Benson CA, Pope RK, Leroux BM, Picel AC, Mateczun AJ, Cilwa KE, Regeimbal JM, Estrella LA, Wolfe DM, Henry MS, Quinones J, Salka S, Bishop-Lilly KA, Young R, Hamilton T. 2017. Development and use of personalized bacteriophage-based therapeutic cocktails to treat a patient with a disseminated resistant Acinetobacter baumannii Infection. Antimicrob Agents Chemother 61.

5. Scholl D. 2017. Phage tail-like bacteriocins. Annu Rev Virol 4:453-467.

6. Scholl D, Martin DW, Jr. 2008. Antibacterial efficacy of R-type pyocins towards Pseudomonas aeruginosa in a murine peritonitis model. Antimicrob Agents Chemother 52:1647-52.

7. Gebhart D, Lok S, Clare S, Tomas M, Stares M, Scholl D, Donskey CJ, Lawley TD, Govoni GR. 2015. A modified R-type bacteriocin specifically targeting Clostridium difficile prevents colonization of mice without affecting gut microbiota diversity. MBio 6 .

8. Scholl D, Cooley M, Williams SR, Gebhart D, Martin D, Bates A, Mandrell R. 2009. An engineered R-type pyocin is a highly specific and sensitive bactericidal agent for the food-borne pathogen Escherichia coli O157:H7. Antimicrob Agents Chemother 53:307480 .

9. Ritchie JM, Greenwich JL, Davis BM, Bronson RT, Gebhart D, Williams SR, Martin D, Scholl D, Waldor MK. 2011. An Escherichia coli O157-specific engineered pyocin prevents and ameliorates infection by E. coli $\mathrm{O} 157: \mathrm{H} 7$ in an animal model of diarrheal disease. Antimicrob Agents Chemother 55:5469-74.

10. Lee G, Chakraborty U, Gebhart D, Govoni GR, Zhou ZH, Scholl D. 2016. F-Type Bacteriocins of Listeria monocytogenes: a new class of phage tail-like structures reveals 
broad parallel coevolution between tailed bacteriophages and high-molecular-weight bacteriocins. J Bacteriol 198:2784-93.

11. Michel-Briand Y, Baysse C. 2002. The pyocins of Pseudomonas aeruginosa. Biochimie 84:499-510.

12. Nakayama K, Takashima K, Ishihara H, Shinomiya T, Kageyama M, Kanaya S, Ohnishi M, Murata T, Mori H, Hayashi T. 2000. The R-type pyocin of Pseudomonas aeruginosa is related to P2 phage, and the F-type is related to lambda phage. Mol Microbiol 38:21331.

13. Uratani Y, Hoshino T. 1984. Pyocin R1 inhibits active transport in Pseudomonas aeruginosa and depolarizes membrane potential. J Bacteriol 157:632-6.

14. Scholl D, Gebhart D, Williams SR, Bates A, Mandrell R. 2012. Genome sequence of $E$. coli O104:H4 leads to rapid development of a targeted antimicrobial agent against this emerging pathogen. PLoS One 7:e33637.

15. Williams SR, Gebhart D, Martin DW, Scholl D. 2008. Retargeting R-type pyocins to generate novel bactericidal protein complexes. Appl Environ Microbiol 74:3868-76.

16. Ghequire MG, De Mot R. 2014. Ribosomally encoded antibacterial proteins and peptides from Pseudomonas. FEMS Microbiol Rev 38:523-68.

17. Kuroda K, Kageyama M. 1981. Comparative study of F-type pyocins of Pseudomonas aeruginosa. J Biochem 89:1721-36.

18. Govan JR. 1974. Studies on the pyocins of Pseudomonas aeruginosa: production of contractile and flexuous pyocins in Pseudomonas aeruginosa. J Gen Microbiol 80:17-30.

19. Kuroda K, Kageyama M. 1979. Biochemical properties of a new flexuous bacteriocin, pyocin F1, produced by Pseudomonas aeruginosa. J Biochem 85:7-19.

20. Takeya K, Minamishima Y, Amako K, Ohnishi Y. 1967. A small rod-shaped pyocin. Virology 31:166-8.

21. Bondy-Denomy J, Qian J, Westra ER, Buckling A, Guttman DS, Davidson AR, Maxwell KL. 2016. Prophages mediate defense against phage infection through diverse mechanisms. ISME J doi:10.1038/ismej.2016.79.

22. Matsui H, Sano Y, Ishihara H, Shinomiya T. 1993. Regulation of pyocin genes in Pseudomonas aeruginosa by positive (prtN) and negative (prtR) regulatory genes. $\mathrm{J}$ Bacteriol 175:1257-63.

23. Winsor GL, Lam DK, Fleming L, Lo R, Whiteside MD, Yu NY, Hancock RE, Brinkman FS. 2011. Pseudomonas Genome Database: improved comparative analysis and population genomics capability for Pseudomonas genomes. Nucleic Acids Res 39:D596600.

24. Deighan P, Hochschild A. 2007. The bacteriophage lambdaQ anti-terminator protein regulates late gene expression as a stable component of the transcription elongation complex. Mol Microbiol 63:911-20.

25. Young R. 2014. Phage lysis: three steps, three choices, one outcome. J Microbiol 52:24358 .

26. Kohler T, Donner V, van Delden C. 2010. Lipopolysaccharide as shield and receptor for R-pyocin-mediated killing in Pseudomonas aeruginosa. J Bacteriol 192:1921-8.

27. Davidson AR, Cardarelli L, Pell LG, Radford DR, Maxwell KL. 2012. Long noncontractile tail machines of bacteriophages. Adv Exp Med Biol 726:115-42.

28. Pell LG, Liu A, Edmonds L, Donaldson LW, Howell PL, Davidson AR. 2009. The X-ray crystal structure of the phage lambda tail terminator protein reveals the biologically 
relevant hexameric ring structure and demonstrates a conserved mechanism of tail termination among diverse long-tailed phages. J Mol Biol 389:938-51.

29. Auzat I, Petitpas I, Lurz R, Weise F, Tavares P. 2014. A touch of glue to complete bacteriophage assembly: the tail-to-head joining protein (THJP) family. Mol Microbiol 91:1164-78.

30. Wang J, Hofnung M, Charbit A. 2000. The C-terminal portion of the tail fiber protein of bacteriophage lambda is responsible for binding to LamB, its receptor at the surface of Escherichia coli K-12. J Bacteriol 182:508-12.

31. Werts C, Michel V, Hofnung M, Charbit A. 1994. Adsorption of bacteriophage lambda on the LamB protein of Escherichia coli $\mathrm{K}-12$ : point mutations in gene $\mathrm{J}$ of lambda responsible for extended host range. J Bacteriol 176:941-7.

32. Finn RD, Clements J, Arndt W, Miller BL, Wheeler TJ, Schreiber F, Bateman A, Eddy SR. 2015. HMMER web server: 2015 update. Nucleic Acids Res 43:W30-8.

33. Hendrix RW, Duda RL. 1992. Bacteriophage lambda PaPa: not the mother of all lambda phages. Science 258:1145-8.

34. North OI, Sakai K, Yamashita E, Nakagawa A, Iwazaki T, Buttner CR, Takeda S, Davidson AR. 2019. Phage tail fibre assembly proteins employ a modular structure to drive the correct folding of diverse fibres. Nat Microbiol 4:1645-1653.

35. Gillies RR, Govan JR. 1966. Typing of Pseudomonas pyocyanea by pyocine production. J Pathol Bacteriol 91:339-45.

36. Liberati NT, Urbach JM, Miyata S, Lee DG, Drenkard E, Wu G, Villanueva J, Wei T, Ausubel FM. 2006. An ordered, nonredundant library of Pseudomonas aeruginosa strain PA14 transposon insertion mutants. Proc Natl Acad Sci U S A 103:2833-8.

37. Lam JS, Taylor VL, Islam ST, Hao Y, Kocincova D. 2011. Genetic and Functional Diversity of Pseudomonas aeruginosa Lipopolysaccharide. Front Microbiol 2:118.

38. Zerbino DR, Birney E. 2008. Velvet: algorithms for de novo short read assembly using de Bruijn graphs. Genome Res 18:821-9.

39. Kearse M, Moir R, Wilson A, Stones-Havas S, Cheung M, Sturrock S, Buxton S, Cooper A, Markowitz S, Duran C, Thierer T, Ashton B, Meintjes P, Drummond A. 2012. Geneious Basic: an integrated and extendable desktop software platform for the organization and analysis of sequence data. Bioinformatics 28:1647-9.

40. Altschul SF, Koonin EV. 1998. Iterated profile searches with PSI-BLAST--a tool for discovery in protein databases. Trends Biochem Sci 23:444-7.

41. Waterhouse AM, Procter JB, Martin DM, Clamp M, Barton GJ. 2009. Jalview Version 2-a multiple sequence alignment editor and analysis workbench. Bioinformatics 25:118991.

42. Zimmermann L, Stephens A, Nam SZ, Rau D, Kubler J, Lozajic M, Gabler F, Soding J, Lupas AN, Alva V. 2018. A completely reimplemented MPI bioinformatics toolkit with a new HHpred server at its core. J Mol Biol 430:2237-2243.

43. Finn RD, Mistry J, Tate J, Coggill P, Heger A, Pollington JE, Gavin OL, Gunasekaran P, Ceric G, Forslund K, Holm L, Sonnhammer EL, Eddy SR, Bateman A. 2010. The Pfam protein families database. Nucleic Acids Res 38:D211-22.

44. Buttner CR, Wu Y, Maxwell KL, Davidson AR. 2016. Baseplate assembly of phage Mu: Defining the conserved core components of contractile-tailed phages and related bacterial systems. Proc Natl Acad Sci U S A 113:10174-9. 
bioRxiv preprint doi: https://doi.org/10.1101/2021.02 16.431561; this version posted February 17, 2021. The copyright holder for this preprint

(which was not certified by peer review) is the author/funder, who has granted bioRxiv a license to display the preprint in perpetuity. It is made available under aCC-BY 4.0 International license.

45. Xu J, Hendrix RW, Duda RL. 2014. Chaperone-protein interactions that mediate assembly of the bacteriophage lambda tail to the correct length. J Mol Biol 426:1004-18. 


\section{TABLES}

633 Table 1. Gene composition of the pyocin operon in P. aeruginosa strain PA14

\begin{tabular}{|c|c|c|c|}
\hline $\begin{array}{l}\text { Gene name } \\
\text { (this study) }\end{array}$ & PA14 locus-tag & $\begin{array}{l}\text { Identity with } \\
\text { HK022 } \\
\text { Homolog }(\%)^{1}\end{array}$ & Protein Function \\
\hline pyoRF1 & PA14_07950 & & Activator (PrtN) \\
\hline pyoRF2 & PA14_07960 & & Repressor (PrtR) \\
\hline pyoRF3 & PA14_07970 & & Zinc finger transcription factor ${ }^{2}$ \\
\hline pyoRF4 & PA14_07980 & & Lambda gpQ-like ${ }^{2}$ \\
\hline pyoRF5 & PA14_07990 & & Holin $^{2}$ \\
\hline pyoR1 & PA14_08000 & & Tail Terminator $^{2}$ \\
\hline pyoR2 & PA14_08010 & & Tail Spike \\
\hline pyoR3 & PA14_08020 & & Baseplate Wedge $1^{3}$ \\
\hline pyoR4 & PA14_08030 & & Baseplate Wedge $2^{3}$ \\
\hline pyoR5 & PA14_08040 & & Baseplate Wedge $3^{3}$ \\
\hline pyoR6 & PA14_08050 & & Tail Fiber \\
\hline pyoR7 & PA14_08060 & & Tail Fiber Chaperone \\
\hline pyoR8 & PA14_08070 & & Tail Sheath \\
\hline pyoR9 & PA14_08090 & & Tail Tube \\
\hline pyoR10 & PA14_08100 & & Tape Measure Chaperone \\
\hline pyoR11 & PA14_08110 & & Tape Measure Chaperone ${ }^{4}$ \\
\hline pyoR12 & PA14_08120 & & Tape Measure \\
\hline pyoR13 & PA14_08130 & & Baseplate hub $1^{3}$ \\
\hline pyoR14 & PA14_08140 & & Baseplate LysM domain \\
\hline pyoR15 & PA14_08150 & & Baseplate hub $2^{3}$ \\
\hline pyoRF6 & PA14_08160 & & Peptidoglycan hydrolase \\
\hline pyoRF7 & PA14_08180 & & Spannin $(\mathrm{Rz})^{2}$ \\
\hline pyoRF8 & PA14_08190 & & Spannin $(\mathrm{Rz} 1)^{2}$ \\
\hline pyoF1 & PA14_08200 & & Unknown \\
\hline pyoF2 & PA14_08210 & 54 & Tail Tube \\
\hline pyoF3 & PA14_08220 & ND & Tape Measure Chaperone \\
\hline pyoF4 & PA14_08230 & 36 & Tape Measure Chaperone ${ }^{4}$ \\
\hline pyoF5 & PA14_08240 & 35 & Tape Measure \\
\hline pyoF6 & PA14_08250 & 37 & Tail tip protein \\
\hline pyoF7 & PA14_08260 & 46 & Tail tip protein \\
\hline pyoF 8 & PA14_08270 & 44 & Tail tip protein \\
\hline pyoF9 & PA14_08280 & 49 & Tail tip protein \\
\hline pyoF 10 & PA14_08300 & 44 & Central fiber \\
\hline
\end{tabular}




\begin{tabular}{lccl} 
pyoF11 & 5 & 24 & Module 1 specificity determinant \\
pyoF12 & NA & ND & Module 1 specificity determinant \\
pyoF13 & NA & 20 & Module 2 specificity determinant \\
pyoF14 & PA14_08310 & & Module 2 specificity determinant \\
pyoF15 & PA14_08320 & & Module 2 specificity determinant \\
\hline
\end{tabular}

$635{ }^{1}$ Values are pairwise percent amino acid identity, and are shown for tail morphogenesis proteins that are

636 present in both E. coli phage HK022 and the F-type pyocin cluster. The genes encoding these proteins are

637 arranged syntenically in the two clusters. "ND" denotes that likely homologous proteins were present, but

638 the pairwise sequence identity was below $20 \%$.

$639{ }^{2}$ Functions were predicted using HHpred.

$640{ }^{3}$ These myophage baseplate functions are defined in Buttner et al. (44).

$641{ }^{4}$ The second Tape Measure Chaperone encoding segment is appended to the first through a programmed

642 translational frameshift (45). The annotation of this ORF is missed in many P. aeruginosa genomes.

$643{ }^{5}$ These proteins, though clearly encoded in the PA14 genome, were not annotated. 
646 Table 2. Plasmid-based complementation of pyoF11 to pyoF15 mutants

\begin{tabular}{|c|c|c|}
\hline $\begin{array}{l}\text { Mutated } \\
\text { Gene }\end{array}$ & $\begin{array}{c}\text { Gene(s) expressed on } \\
\text { complementing plasmid }\end{array}$ & Complementation Result \\
\hline pyoF11 & pyoF11 & No \\
\hline pyoF11 & pyoF $11+$ pyoF 12 & Yes \\
\hline pyoF11 & $\Delta p y o F 11^{1}+$ pyoF 12 & No \\
\hline pyoF11 & pyoF 12 & No \\
\hline pyoF12 & pyoF12 & Yes \\
\hline pyoF13 & pyoF13 & No \\
\hline pyoF13 & pyoF $13+$ pyoF $14+$ pyoF 15 & Yes \\
\hline pyoF13 & pyoF $14+$ pyoF 15 & No \\
\hline pyoFl4 & pyoF14 & No \\
\hline pyoFl4 & pyoF $14+$ pyoF 15 & Yes \\
\hline pyoF14 & $\Delta p y o F 14^{1}+$ pyoF 15 & No \\
\hline pyoF15 & pyoF15 & Yes \\
\hline pyoF15 & $\Delta p y o F 14^{1}+$ pyoF 15 & Yes \\
\hline
\end{tabular}

$647{ }^{1}$ These plasmids express two genes but the first bears an in-frame deletion. 


\section{FIGURE LEGENDS}

649 FIG 1 Transmission electron micrographs of lysates of cells producing R- and F-type pyocins.

650 Shown are a lysate of strain S22 (left panel), which produces both R- and F-type pyocins; a

651 lysate of strain S13 (middle panel), which produces just R-pyocins; and a lysate of strain S18

652 (right panel), which produces just F-pyocins. R-type pyocin particles are indicated by green

653 arrows and F-type pyocin particles are indicated by red arrows. Grids were negatively stained

654 with uranyl acetate. The scale bar shown applies to all three micrographs.

FIG 2 R- and F-type pyocin operons. (a) Three types of R- or F-pyocin operons are found in $P$. aeruginosa: operons encode just R-pyocins (top), R- and F-pyocins (middle, or just F-pyocins (bottom). All three types share the same regulatory genes (orange) and lysis genes (brown). three types of operons are located in the same position in the P. aeruginosa genome between the $\operatorname{trp} E$ and $\operatorname{trp} G$ genes. (b) A close up of genes encoded at the 3'-end of the F-type pyocin clusters

662 show the six different groups identified in our sequenced strains. The numbers above the genes

663 indicate the percent pairwise sequence identity of the encoded protein with the homolog found in 664 strain PA14 (group 5). Proteins PyoF13, PyoF14 and PyoF15, which are duplicated in group F2, 665 are very closely related (>95\% sequence identity) in groups F5, F4 and F2 (second group) as 666 indicated by their coloring. The same proteins are highly similar in groups F1 and F6. In the 667 cases of PyoF10 and PyoF13, sequence comparison were performed including only their variable

668 C-terminal domains. P. aeruginosa strains where certain groups were previously identified are 669 shown in parentheses.

670 FIG 3 Bactericidal activity of F-type pyocins. (a) Bactericidal activity caused by F- or R-type 671 pyocins can be distinguished from that caused by phages of S-pyocins. S-pyocin activity is 672 destroyed by addition of proteinase K. Zones of clearing resulting from phages resolve into 673 individual plaques upon dilution. (b) The bactericidal activity of F-type pyocins on a selected 674 group of bacterial lawns is shown. These lawns were selected to emphasize the differences in 675 specificity among the different groups. Black boxes denote strains killed by a given F-type 676 pyocin while white boxes denote no killing. The gray box indicates a case where the killing by 677 pyocins was occluded by phage plaquing. Bactericidal activity of the F5 group was determined using a mutant strain of PA14 bearing a transposon insertion in the pyoR3 gene, so that the lysate 
679 contained only F-type pyocin particles (indicated by an asterisk). The F1- and F6-type pyocins

680 were produced in strains that also produced R1-pyocins. By comparing with a strain producing

681 only R1-type pyocins, two strains killed only by these F-type pyocins could be identified

682 (marked with "F"). (c) R- or F-pyocin lysates made from strain PA14 were spotted on a lawn of

683 strain S19. Lysates were produced from wild-type PA14 and strains bearing transposon insertions

684 in either the pyoR6 or pyoF10 genes. (d) F7-type pyocins produced from strain S24 or Strain S8

685 were spotted on lawns of strain S14 or strain PAO1.

686

687 FIG 4 (a) The effect of LPS serotype on bactericidal activity of F-type pyocins. The bactericidal 688 activity of F-type pyocins on a group of bacterial lawns arranged by their serotypes is shown.

689 Black boxes denote strains killed by a given F-type pyocin while white boxes denote no killing.

690 The gray box indicates a case where the killing by pyocins was occluded by phage plaquing.

691 Bactericidal activity of the F5 group was determined using a mutant strain of PA14 bearing a

692 transposon insertion in the pyoR3 gene, so that the lysate contained only F-type pyocin particles

693 (indicated by an asterisk). The F1- and F6-type pyocins were produced in strains that also

694 produced R1-pyocins. By comparing with a strain producing only R1-type pyocins, two strains

695 killed only by these F-type pyocins could be identified (marked with "F"). (b) The indicated F-

696 type pyocin containing lysates were tested against mutants that lack OSA $(\Delta w b p M)$.

698 FIG 5 All F-type pyocin groups. A close up of genes encoded at the 3'-end of F-type pyocin 699 clusters shows the 11 different groups identified in our sequenced strains and in the database.

700 Groups of genes are colored the same if the proteins they encode display greater than $90 \%$

701 sequence identity. The extent of Specificity Modules 1 and 2 are shown at the top as are the

702 names of the genes in these regions. P. aeruginosa strains where certain groups were previously

703 identified are shown in parentheses. 


\section{Supplemental Materials and Methods}

707 In separate file.

\section{Supplementary Table 1}

709 List of strains used and origin (in separate file)

\section{Supplementary Table 2}

711 List of primers used (in separate file)

714 FIG S1 Pairwise identities among F-type pyocin proteins encoded at the 3'-end of the operon.

715 All against all pairwise percent identities for the indicated proteins are shown. The F-pyocin

716 groups of the proteins that are being compared are indicated at the sides and top of each table.

717 Numbers that are shaded denote groups that share Specificity Modules. The PyoF10 comparisons

718 include only the C-terminal 60 amino acids. PyoF13 comparisons include only the last 210

719 residues of these proteins. The F7 group does not encode PyoF14, so these boxes are left blank.

720 "<20" denotes sequences that could not be well aligned in a pairwise alignment. F21 and F22

721 refer to the duplicated PyoF13, PyoF14, and PyoF15 proteins encoded in the F2 group.

723 FIG S2 Protein sequence alignments of PyoF11 and PyoF12 from each F-type pyocin group. (a)

724 An alignment of PyoF11 homologs is shown from the $11 \mathrm{~F}$-type pyocin groups and selected

725 phages. (b) An alignment of PyoF11 homologs is shown from the 11 F-type pyocin groups and

726 selected phages. The phage proteins are from Burkholderia phages KS9 (NC_013055) and

727 BcepGomr (NC_009447); P. aeruginosa phages LIT1 (NC_013692) and LUZ7 (NC_013691);

728 and E. coli phages T1 (NC_005833) and N15 (NC_001901).

730 FIG S3 Protein sequence alignments of PyoF13 from each F-type pyocin group. (a) An

731 alignment of the N-terminal 140 amino acids of PyoF13 homologs from the 11 F-type pyocin 
732 groups is shown. (b) An alignment of the C-terminal 210 amino acids of PyoF13 homologs from 733 the $11 \mathrm{~F}$-type pyocin groups is shown.

734

735 FIG S4 Protein sequence alignments of the tail fiber proteins (PyoR6) from each R-type pyocin

736 group. (a) An alignment of the N-terminal 450 amino acids of the PyoR6 homologs is shown. (b)

737 An alignment of the C-terminal 250 amino acids PyoR6 homologs is shown. (c) The pairwise

738 sequence identities of the PyoR6 N-terminal and C-terminal regions are shown. The locus tags

739 for the proteins shown are R1 (PLES_06171), R2 (PA14_08050), and R5 (PA0620).

741 FIG S5 The bactericidal activity of all F-type pyocin lysates tested on all of the P. aeruginosa

742 strains used in this study. 

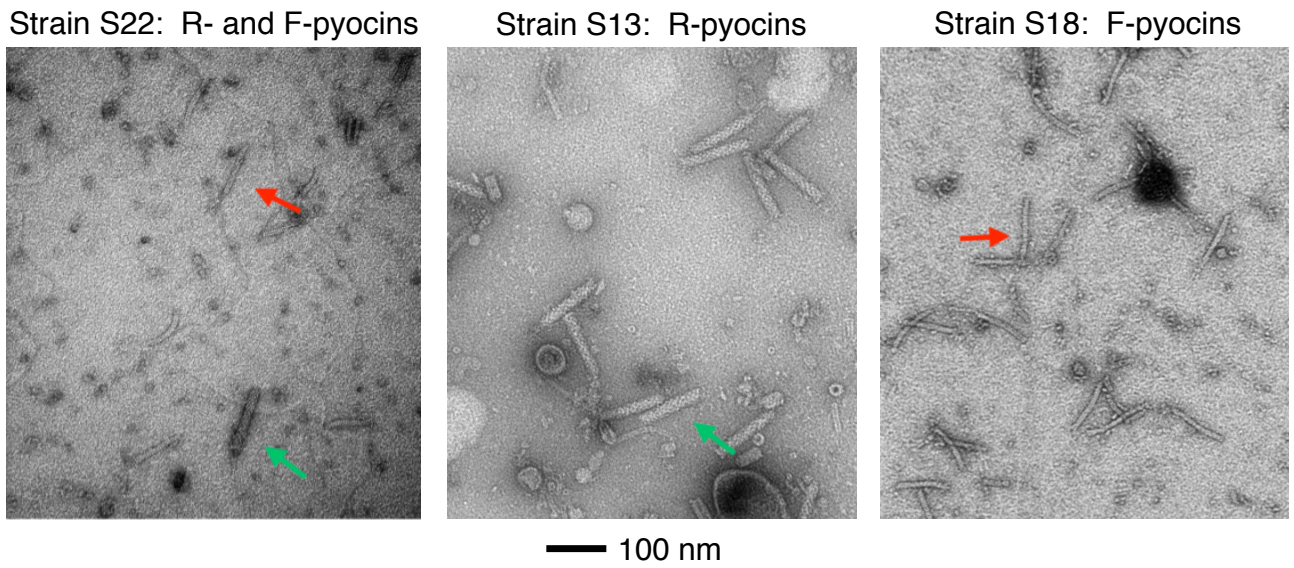

FIG 1 Transmission electron micrographs of lysates of cells producing R- and F-type pyocins. Shown are a lysate of strain S22 (left panel), which produces both R- and F-type pyocins; a lysate of strain S13 (middle panel), which produces just R-pyocins; and a lysate of strain S18 (right panel), which produces just F-pyocins. R-type pyocin particles are indicated by green arrows and F-type pyocin particles are indicated by red arrows. Grids were negatively stained with uranyl acetate. The scale bar shown applies to all three micrographs. 
a

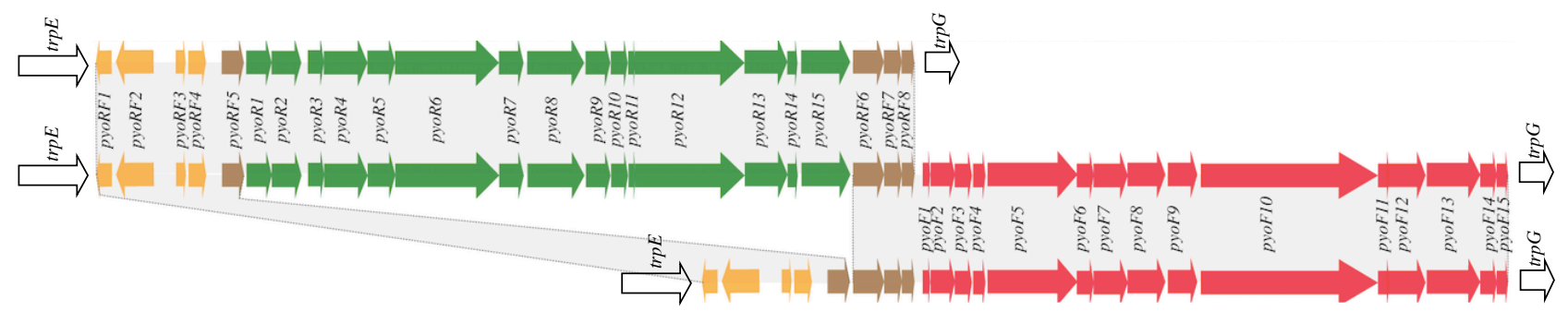

b

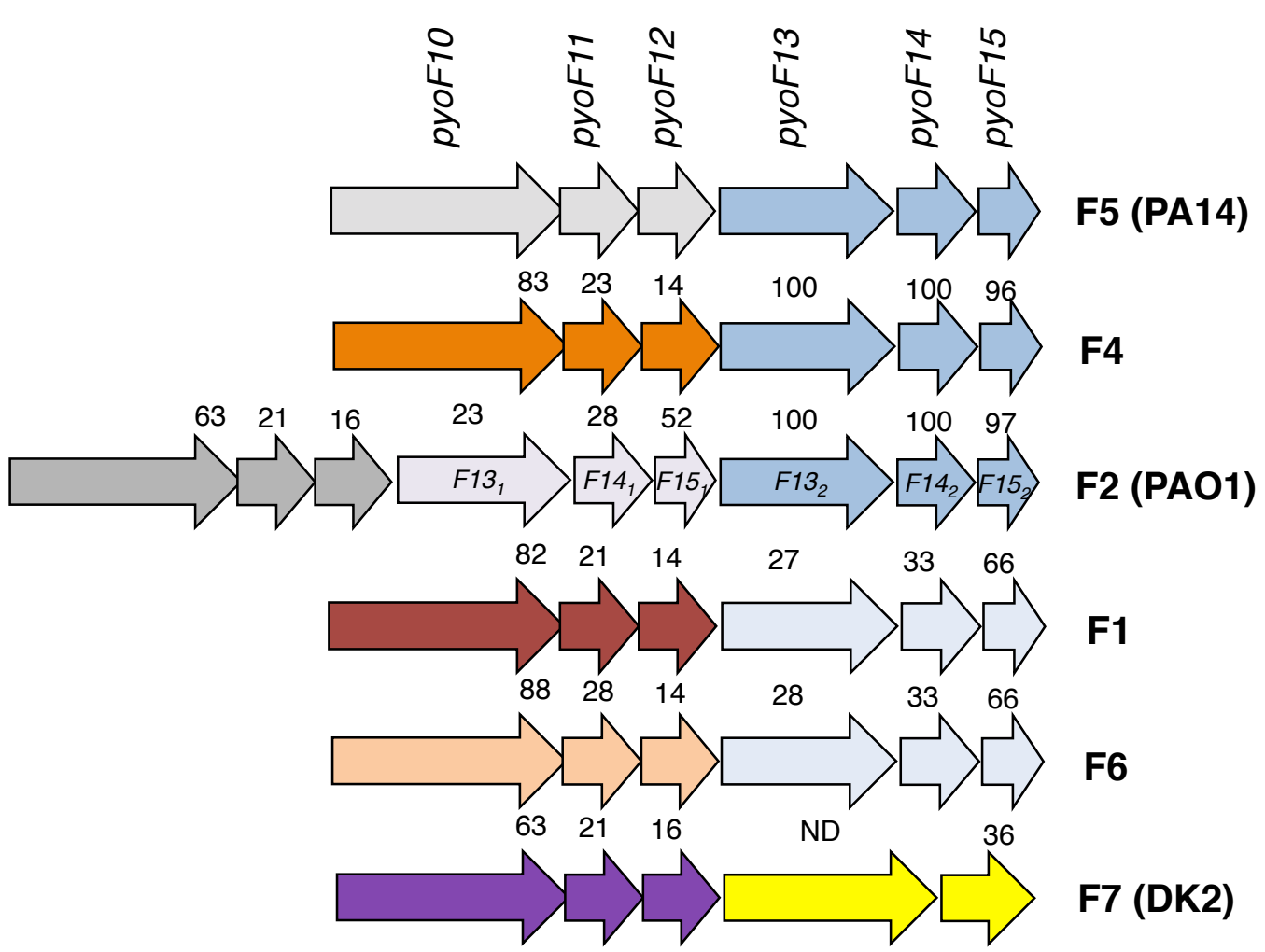

FIG 2 R-and F-type pyocin operons. (a) Three types of R- or F-pyocin operons are found in $P$. aeruginosa: operons encode just R-pyocins (top), R- and F-pyocins (middle, or just F-pyocins (bottom). All three types share the same regulatory genes (orange) and lysis genes (brown). Genes unique to R-pyocins are colored green and those unique to F-pyocins are colored red. All three types of operons are located in the same position in the P. aeruginosa genome between the trpE and $\operatorname{trp} G$ genes. (b) A close up of genes encoded at the 3'-end of the F-type pyocin clusters show the six different groups identified in our sequenced strains. The numbers above the genes indicate the percent pairwise sequence identity of the encoded protein with the homolog found in strain PA14 (group 5). Proteins PyoF13, PyoF14 and PyoF15, which are duplicated in group F2, are very closely related ( $>95 \%$ sequence identity) in groups F5, F4 and F2 (second group) as indicated by their coloring. The same proteins are highly similar in groups F1 and F6. In the cases of PyoF10 and PyoF13, sequence comparison were carried only including their variable Cterminal domains. P. aeruginosa strains where certain groups were previously identified are shown in parentheses. 
a

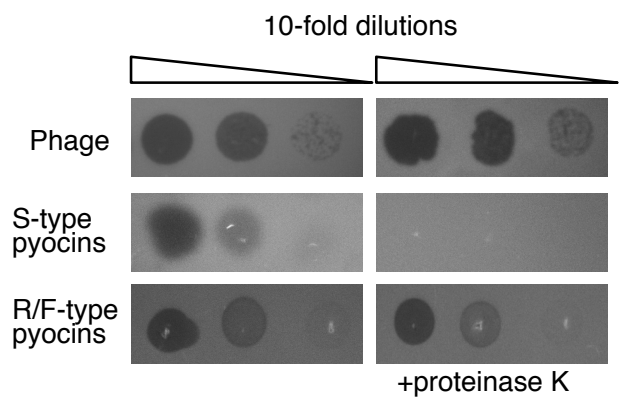

C

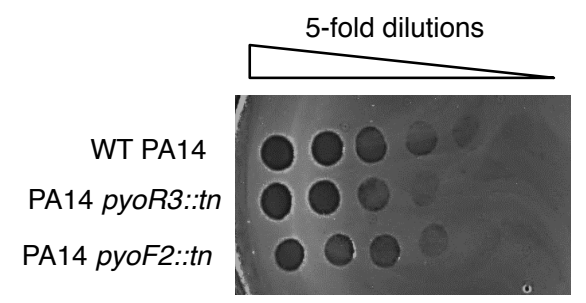

d

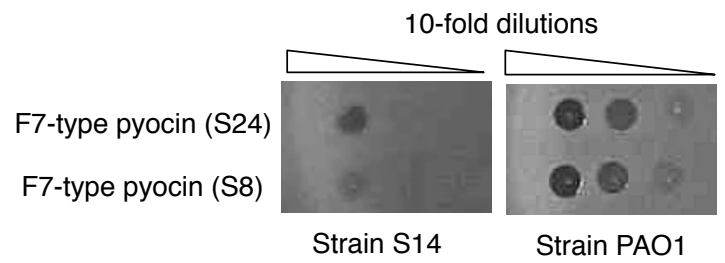

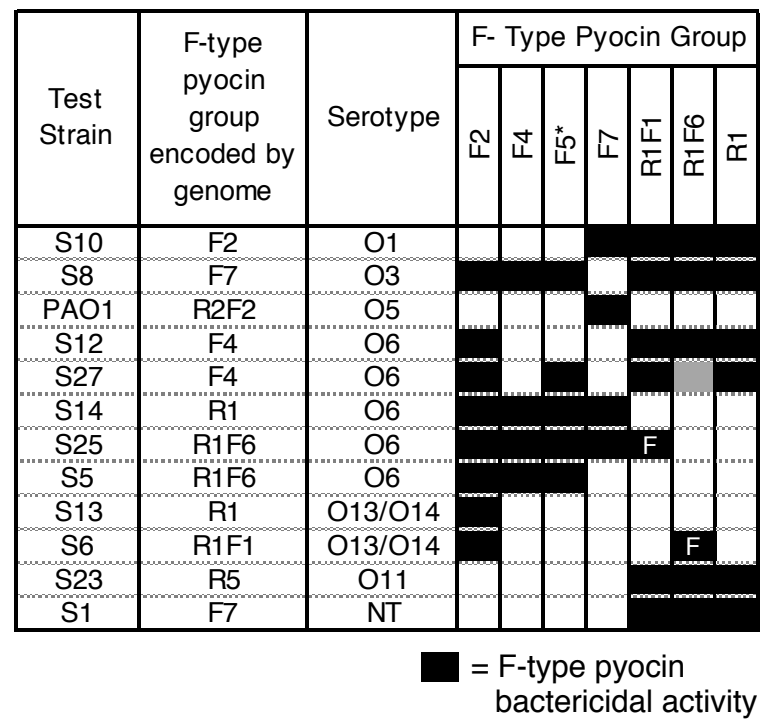

bactericidal activity

FIG 3 Bactericidal activity of F-type pyocins. (a) Bactericidal activity caused by F- or R-type pyocins can be distinguished from that caused by phages of S-pyocins. S-pyocin activity is destroyed by addition of proteinase $\mathrm{K}$. Zones of clearing resulting from phages resolve into individual plaques upon dilution. (b) The bactericidal activity of F-type pyocins on a selected group of bacterial lawns is shown. These lawns were selected to emphasize the differences in specificity among the different groups. Black boxes denote strains killed by a given F-type pyocin while white boxes denote no killing. The gray box indicates a case where the killing by pyocins was occluded by phage plaquing. Bactericidal activity of the F5 group was determined using a mutant strain of PA14 bearing a transposon insertion in the pyoR3 gene, so that the lysate contained only F-type pyocin particles (indicated by an asterisk). The F1- and F6-type pyocins were produced in strains that also produced R1-pyocins. By comparing with a strain producing only R1-type pyocins, two strains killed only by these F-type pyocins could be identified (marked with "F"). (c) R- or F-pyocin lysates made from strain PA14 were spotted on a lawn of strain S19. Lysates were produced from wild-type PA14 and strains bearing transposon insertions in either the pyoR6 or pyoF 10 genes. (d) F7-type pyocins produced from strain S24 or Strain S8 were spotted on lawns of strain $\mathrm{S} 14$ or strain PAO1. 


\begin{tabular}{|c|c|c|c|c|c|c|c|c|c|}
\hline \multirow[b]{2}{*}{$\begin{array}{l}\text { Test } \\
\text { Strain }\end{array}$} & \multirow{2}{*}{$\begin{array}{c}\text { F-type } \\
\text { pyocin } \\
\text { group } \\
\text { encoded by } \\
\text { genome }\end{array}$} & \multirow[b]{2}{*}{ Serotype } & \multicolumn{7}{|c|}{ pyocin group } \\
\hline & & & $\mathbb{\Psi}$ & 过 & $\stackrel{*}{*}$ & $\mathbb{4}$ & $\frac{\bar{L}}{\bar{x}}$ & 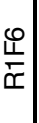 & $\bar{\simeq}$ \\
\hline $\mathrm{S} 10$ & F2 & 01 & & & & & & & \\
\hline S28 & F2 & 01 & & & & & & & \\
\hline S3 & R2 F2 & $\mathrm{O} 2$ & & & & & & & \\
\hline S4 & R2 F2 & $\mathrm{O} 2$ & & & & & & & \\
\hline S7 & R2 F2 & $\mathrm{O} 2$ & & & & & & & \\
\hline S8 & F8 & 03 & & & & & & & \\
\hline S24 & F8 & $\mathrm{O} 3$ & & & & & & & \\
\hline S15 & R5 & 04 & & & & & & & \\
\hline PAO1 & R2 F2 & 05 & & & & & & & \\
\hline S9 & R2 F2 & O5 & & & & & & & \\
\hline $\mathrm{S} 17$ & R2 F2 & O5 & & & & & & & \\
\hline S11 & $\mathrm{R} 2 \mathrm{~F} 2$ & O5 & & & & & & & \\
\hline S12 & $\mathrm{F} 4$ & 06 & & & & & & & \\
\hline S27 & F4 & O6 & & & & & & & \\
\hline $\mathrm{S} 14$ & $\mathrm{R} 1$ & O6 & & & & & & & \\
\hline S22 & $\mathrm{R} 1$ & O6 & & & & & & & \\
\hline S19 & $\mathrm{R} 1$ & $\mathrm{O} 6$ & & & & & & & \\
\hline S20 & $\mathrm{R} 1$ & O6 & & & & & & & \\
\hline S25 & R1 F6 & O6 & & & & & $\mathrm{F}$ & & \\
\hline S5 & R1 F6 & O6 & & & & & & & \\
\hline PA14 & R2 F5 & 010 & & & & & & & \\
\hline S16 & R2 F5 & 010 & & & & & & & \\
\hline S13 & $\mathrm{R} 1$ & 013/014 & & & & & & & \\
\hline S21 & R1 & $013 / 014$ & & & & & & & \\
\hline S6 & $\mathrm{R} 1 \mathrm{~F} 1$ & $013 / 014$ & & & & & & $\mathrm{~F}$ & \\
\hline S18 & R2 F2 & 016 & & & & & & & \\
\hline S2 & $\mathrm{R} 1$ & HOMMA15 & & & & & & & \\
\hline S23 & R5 & 011 & & & & & & & \\
\hline S1 & F7 & NT & & & & & & & \\
\hline S26 & F7 & NT & & & & & & & \\
\hline
\end{tabular}

b

\begin{tabular}{|c|c|c|c|c|c|c|c|c|c|}
\hline \multirow[b]{2}{*}{ Test Strain } & \multirow{2}{*}{$\begin{array}{c}\text { F-type } \\
\text { pyocin } \\
\text { group } \\
\text { encoded by } \\
\text { genome }\end{array}$} & \multirow[b]{2}{*}{ Serotype } & \multicolumn{7}{|c|}{ pyocin group } \\
\hline & & & $\mathbb{\Psi}$ & 过 & 点 & 논 & $\frac{\overline{4}}{\bar{x}}$ & $\frac{\stackrel{0}{\mathbb{1}}}{\underline{\sim}}$ & $\bar{x}$ \\
\hline $\begin{array}{c}\text { PAO1 } \\
\text { PAO1 } \triangle w b p M\end{array}$ & $\begin{array}{l}\text { R2 F2 } \\
\text { R2 F2 }\end{array}$ & $\begin{array}{c}\text { O5 } \\
\text { no OSA }\end{array}$ & & & & & & & \\
\hline $\begin{array}{c}\mathrm{PA} 14 \\
\mathrm{PA} 14 \Delta w b p M\end{array}$ & $\begin{array}{l}\text { R2 F5 } \\
\text { R2 F5 }\end{array}$ & $\begin{array}{c}\text { O10 } \\
\text { no OSA }\end{array}$ & & & & & & & \\
\hline
\end{tabular}

FIG 4 (a) The effect of LPS serotype on bactericidal activity of F-type pyocins. The bactericidal activity of F-type pyocins on a group of bacterial lawns arranged by their serotypes is shown. Black boxes denote strains killed by a given F-type pyocin while white boxes denote no killing. The gray box indicates a case where the killing by pyocins was occluded by phage plaquing. Bactericidal activity of the F5 group was determined using a mutant strain of PA14 bearing a transposon insertion in the pyoR3 gene, so that the lysate contained only F-type pyocin particles (indicated by an asterisk). The F1- and F6-type pyocins were produced in strains that also produced R1-pyocins. By comparing with a strain producing only R1-type pyocins, two strains killed only by these F-type pyocins could be identified (marked with "F"). (b) The indicated Ftype pyocin containing lysates were tested against mutants that lack OSA $(\Delta w b p M)$. 


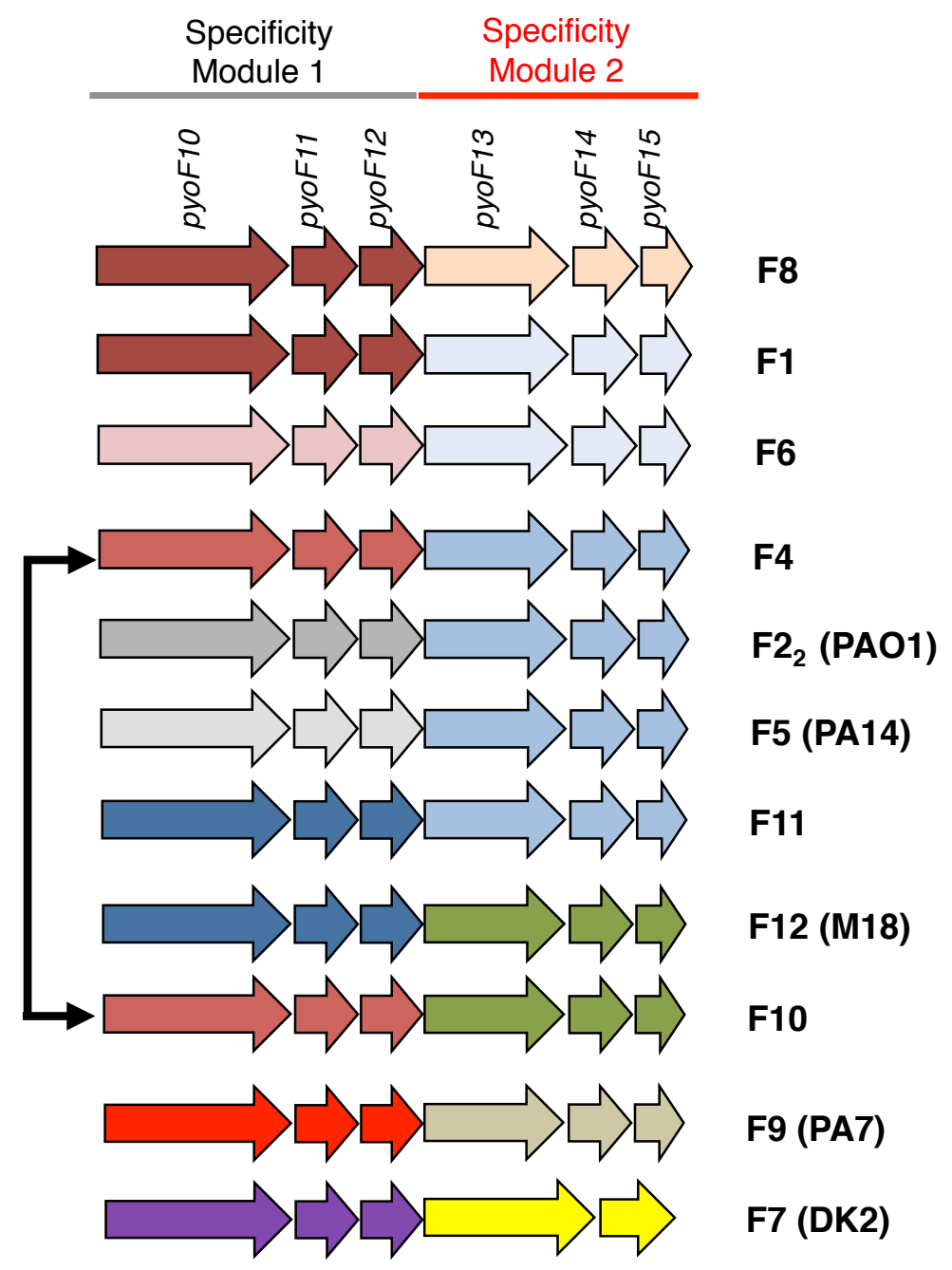

FIG 5 All F-type pyocin groups. A close up of genes encoded at the 3'-end of F-type pyocin clusters shows the 11 different groups identified in our sequenced strains and in the database. Groups of genes are colored the same if the proteins they encode display greater than $90 \%$ sequence identity. The extent of Specificity Modules 1 and 2 are shown at the top as are the names of the genes in these regions. P. aeruginosa strains where certain groups were previously identified are shown in parentheses. 